\title{
Migration of comets to the terrestrial planets
}

\author{
Sergei I. Ipatov ${ }^{1,2}$ and John C. Mather ${ }^{3}$ \\ ${ }^{1}$ Department of Terrestrial Magnetism, Carnegie Institution of Washington \\ Washington DC, USA \\ email: siipatov@hotmail.com \\ http://www.dtm.ciw.edu/ipatov \\ ${ }^{2}$ Space Research Institute, Moscow, Russia \\ ${ }^{3}$ LASP, NASA/Goddard Space Flight Center, Greenbelt, USA \\ email: John.C.Mather@nasa.gov
}

\begin{abstract}
We studied the orbital evolution of objects with initial orbits close to those of Jupiterfamily comets (JFCs), Halley-type comets (HTCs), and long-period comets, and the probabilities of their collisions with the planets. In our runs the probability of a collision of one object with the Earth could be greater than the sum of probabilities for thousands of other objects. Even without the contribution of such a few objects, the probability of a collision of a former JFC with the Earth during the dynamical lifetime of the comet was greater than $4 \times 10^{-6}$. This probability is enough for delivery of all the water to Earth's oceans during the formation of the giant planets. The ratios of probabilities of collisions of JFCs and HTCs with Venus and Mars to the mass of the planet usually were not smaller than that with Earth. Among 30000 considered objects with initial orbits close to those of JFCs, a few objects got Earth-crossing orbits with semimajor axes $a<2 \mathrm{AU}$ and aphelion distances $Q<4.2 \mathrm{AU}$, or even got innerEarth $(Q<0.983 \mathrm{AU})$, Aten, or typical asteroidal orbits, and moved in such orbits for more than $1 \mathrm{Myr}$ (up to tens or even hundreds of Myr). From a dynamical point of view, the fraction of extinct comets among near-Earth objects can exceed several tens of percent, but, probably, many extinct comets disintegrated into mini-comets and dust during a smaller part of their dynamical lifetimes.
\end{abstract}

Keywords. Transport of comets; transport of meteorites

\section{Introduction}

Farinella et al. (1993), Bottke et al. (2002), Binzel et al. (2002), Weissman et al. (2002) believe that asteroids are the main source of near-Earth objects (NEOs). Wetherill (1988) supposed that half of NEOs are former short-period comets. Trans-Neptunian objects (TNOs) can migrate to the near-Earth space. Duncan et al. (1995) and Kuchner et al. (2002) investigated the migration of TNOs to Neptune's orbit, and Levison \& Duncan (1997) studied their migration from Neptune's orbit to Jupiter's orbit. Levison et al. (2006) studied the formation of Encke-type objects. More references on papers devoted to the migration of bodies from different regions of the solar system to the nearEarth space can be found in our previous publications on this problem (Ipatov 1995, 1999, 2000, 2001; Ipatov \& Hahn 1999; Ipatov \& Mather 2003, 2004a,b, 2006a). As the migration of TNOs to Jupiter's orbit was considered by several authors, Ipatov (2002) and Ipatov \& Mather (2003, 2004a,b, 2006a) paid particular attention to the orbital evolution of Jupiter-crossing objects (JCOs), considering a larger number of JCOs than before.

In the present paper, we summarize our studies of migration of cometary objects into NEO orbits, paying particular attention to the probabilities of collision of cometary objects with the terrestrial planets. These studies are based on our previous runs and on 
some new runs. Earlier we did not consider the evolution of orbits of Halley-type comets and long-period comets and did not study the probabilities of collisions of different comets with the giant planets. Though some runs used here are the same as earlier, the discussion on migration of small bodies based on these runs is different.

\section{Initial data}

Ipatov \& Mather (2003, 2004a,b, 2006a) integrated the orbital evolution of about 30000 objects with initial orbits close to those of Jupiter-family comets (JFCs). We considered the gravitational influence of the planets, but omitted the influence of Mercury (except for Comet 2P/Encke) and Pluto. In about a half of the runs we used the method by Bulirsh-Stoer (1966) (BULSTO code), and in other runs we used a symplectic method (RMVS3 code). The integration package of Levison \& Duncan (1994) was used. Usually we investigated the orbital evolution during the dynamical lifetimes of objects (until all the objects reached $2000 \mathrm{AU}$ from the Sun or collided with the Sun).

In the first series of runs (denoted as $n 1$ ) we calculated the orbital evolution of 3100 JCOs moving in initial orbits close to those of 20 real comets with period $5<P_{a}<9$ $\mathrm{yr}$, and in the second series of runs (denoted as $n 2$ ) we considered 13500 initial orbits close to those of 10 real JFCs with period $5<P_{a}<15 \mathrm{yr}$. We selected comets with the above periods among JFCs with numbers between 7 and 75 for $n 1$ and with numbers between 77 and 113 for $n 2$. In other series of runs, initial orbits were close to those of a single comet (2P/Encke, 9P/Tempel 1, 10P/Tempel 2, 22P/Kopff, 28P/Neujmin 1, $39 \mathrm{P} /$ Oterma, or $44 \mathrm{P} /$ Reinmuth 2 ). Comet $2 \mathrm{P} /$ Encke is the only comet with aphelion distance $Q<4.2 \mathrm{AU}$; comets $28 \mathrm{P} /$ Neujmin 1 and 44P/Reinmuth 2 are typical comets with semimajor axis $a \approx 7 \mathrm{AU}$, and other four above comets are typical comets with $a \sim 3-4$ AU. In order to compare the orbital evolution of comets and asteroids, we also studied the orbital evolution of 1300 test asteroids initially moving in the 3:1 and 5:2 resonances with Jupiter.

In our recent runs we also considered objects started from orbits of test long-period comets (initial eccentricity $e_{o}=0.995, q_{o}=a_{o}\left(1-e_{o}\right)=0.9 \mathrm{AU}$ or $q_{o}=0.1 \mathrm{AU}$, initial inclination $i_{o}$ between 0 and $180^{\circ}$ in each run, objects started at perihelion; these runs are denoted as $l p c$ runs) and test Halley-type comets ( $a_{o}=20 \mathrm{AU}, i_{o}$ betwen 0 and $180^{\circ}$ in each run, objects started at perihelion; in some runs $e_{o}=0.975$ and $q_{o}=0.5 \mathrm{AU}$, in other runs $e_{o}=0.9$ and $q_{o}=2 \mathrm{AU}$; these runs are denoted as $h t c$ runs).

In our runs, planets were considered as material points, so literal collisions did not occur. However, using the algorithm suggested by Ipatov $(1988,2000)$ with the correction that takes into account a different velocity at different parts of the orbit (Ipatov \& Mather 2003), and based on all orbital elements sampled with a $500 \mathrm{yr}$ step, we calculated the mean probability $P$ of collisions of migrating objects with a planet. We define $P$ as $P_{\Sigma} / N$, where $P_{\Sigma}$ is the probability for all $N$ objects of a collision of an object with a planet. If it is not mentioned specially, all probabilities below are considered for dynamical lifetimes of objects. Note that our algorithm differed from the Öpik's scheme, and included calculations of a synodic period and the region where the distance between the "first" orbit and the projection of the "second" orbit onto the plane of the "first" orbit is less than the sphere of action (i.e., the Tisserand sphere). The code includes calculations of the probability that both objects are on the same line of sight from the Sun within the above region, of the difference in probabilities for planar and spatial models, and of the probability of a collision inside the sphere.

For BULSTO runs, the integration step error was less than $\epsilon$, where $\epsilon$ varied between $10^{-13}$ and $10^{-8}$ (most of the runs were made for $\epsilon$ equal to $10^{-8}$ and $10^{-9}$ ), and for a 
RMVS3 runs an integration step $d_{s}$ varied from 0.1 to 30 days (most runs were made for $d_{s}=10$ days). In a single run with $N$ (usually $N=250$ ) objects, $\epsilon$ or $d_{s}$ was constant. Results obtained with the use of different methods of integration at different $d_{s}$ or $\epsilon$ were similar (see Ipatov \& Mather 2003 for details), except for probabilities of collisions with the Sun in such runs when this probability was large (for comets $2 \mathrm{P} /$ Encke and 96P/Machholz 1 from $n 2$ series, and the 3:1 resonance with Jupiter). Probabilities of collisions of bodies with planets were close for different integrators even in the latter case because soon after close encounters with the Sun, bodies were ejected into hyperbolic orbits or moved in highly inclined orbits. In most "cometary" runs, the fraction $P_{S u n}$ of comets collided with the Sun was less than $0.02 ; P_{S u n}$ exceeded 0.05 for some $h t c$ runs, and most of objects in $2 \mathrm{P} /$ Encke runs collided with the Sun.

Levison showed that it is difficult to detect solar collisions in any numerical integrator, so he removed objects with perihelion distance $q<q_{\min }$. Our runs were made for direct modeling of collisions with the Sun, but we studied what happens if we consider $q_{\text {min }}$ equal to $k_{S}$ radii $r_{S}$ of the Sun. We obtained that the mean probabilities of collisions of bodies with planets, lifetimes of the objects that spent millions of years in Earth-crossing orbits, and other obtained results were practically the same if we consider that objects disappear when $q$ becomes less than the radius $r_{S}$ of the Sun or even several such radii (i.e., we checked $q<k_{S} \cdot r_{S}$, where $k_{S}$ equals 1,2 , or another value). The only noticeable difference was for Comet $96 \mathrm{P} /$ Machholz 1 from the $n 2$ series, and a smaller one was for Comet $2 \mathrm{P} /$ Encke, but the results of such runs were not included in our statistics. The eccentricity and inclination of Comet 96P/Machholz 1 are large, so usually even for these $n 2$ runs, the collision probabilities of objects with the terrestrial planets were not differed by more than $15 \%$ at $k_{S}=0$ and $k_{S}=1$. Among more than a hundred considered runs, there were three runs, for each of which at $k_{S}=0$ a body in an orbit close to that of Comet $96 \mathrm{P} /$ Machholz 1 was responsible for $70-75 \%$ of collision probabilities with the Earth, and for $k_{S}=1$ a lifetime of such body was much less than for $k_{S}=0$. Nevertheless, for all $\left(\sim 10^{4}\right)$ objects from $n 2$ series, at $k_{S}=0$ the probabilities of collisions with the terrestrial planets were close to those at $k_{S}=1$, even if we consider the above runs. The difference for times spent in Earth-crossing orbits is greater than that for the probabilities and is about $20 \%$. For all runs of the $2 \mathrm{P} /$ Encke series, the difference in time spent in orbits with $Q<4.7 \mathrm{AU}$ for $k_{S}=0$ and for $k_{S}=1$ was less than $4 \%$. In the $2 \mathrm{P} /$ Encke series of runs (and also for the 3:1 resonance with Jupiter), at $k_{S}=0$ we sometimes got orbits with $i>90^{\circ}$, but practically there were no such orbits at $k_{S} \geqslant 1$ (Ipatov \& Mather 2004a,b). Among the objects with initial orbits close to that of Comet $96 \mathrm{P} / \mathrm{Machholz} 1$, we found one object which also got $i>90^{\circ}$ for $3 \mathrm{Myr}$. Inclinations of other such objects did not exceed $90^{\circ}$.

\section{Computer simulations of the migration of comets to near-Earth orbits}

Some migrating JCOs got Earth-crossing orbits. Usually they spent in such orbits only a few thousands years, but a few objects moved in Earth-crossing orbits with aphelion distances $Q<4.2$ AU for millions of years. The total times which 30000 considered objects started from JFC orbits spent in Earth-crossing orbits with $a<2$ AU were due to a few tens of objects, but mainly only to a few of them. In this section we consider only these few objects. With BULSTO at $10^{-9} \leqslant \epsilon \leqslant 10^{-8}$, six and nine objects, respectively from the $10 \mathrm{P} /$ Tempel 2 and $2 \mathrm{P} /$ Encke series, moved into Apollo orbits with $a<2 \mathrm{AU}$ (Al2 orbits) for at least $0.5 \mathrm{Myr}$ each, and five of them remained in such orbits for more than 5 Myr each. Among the JFCs considered with BULSTO, only one and two JFCs 
reached inner-Earth orbits (IEO, $Q<0.983 \mathrm{AU}$ ) and Aten orbits, respectively. Only two objects in series $n 2$ got $A l 2$ orbits during more than 1 Myr. For the $n 1$ series of runs, while moving in JCO orbits, objects had orbital periods $P_{a}<20 \mathrm{yr}$ (JFCs) and $20<P_{a}<200 \mathrm{yr}$ (Halley-type comets) for $32 \%$ and $38 \%$ of the mean value $T_{J}\left(T_{J}=0.12\right.$ Myr) of the total time spent by one object in Jupiter-crossing orbits, respectively.

Four considered former JFCs even got IEO or Aten orbits for Myrs. Note that Ipatov (1995) obtained migration of JCOs into IEO orbits using the method of spheres to consider the gravitational influence of planets. In our BULSTO runs, one former JCO, which had an initial orbit close to that of 10P/Tempel 2, moved in Aten orbits for 3.45 Myr, and the probability of its collision with the Earth from such orbits was 0.344 . It also moved for about $10 \mathrm{Myr}$ in IEO orbits before its collision with Venus, and during this time the probability of its collision with Venus was $P_{V}=0.655$. The above probabilities are greater than the total probabilities for $10^{4}$ other JCOs. Another object (from the 2P BULSTO run) moved in highly eccentric Aten orbits for $83 \mathrm{Myr}$, and its lifetime before collision with the Sun was 352 Myr. Its probability of collisions with Earth, Venus, and Mars during its lifetime was $0.172,0.224$, and 0.065 , respectively. With RMVS3 at $d_{s} \leqslant 10$ days for the $2 \mathrm{P}$ run, the probability of collisions with Earth for one object was greater by a factor of 30 than for 250 other objects. For series $n 1$ with RMVS3, the probability of a collision with the Earth for one object with an initial orbit close to that of Comet 44P/Reinmuth 2 was $88 \%$ of the total probability for 1200 objects from this series, and the total probability for 1198 objects was only $4 \%$. For series $44 \mathrm{P} /$ Reinmuth 2 with $N=1500$ there were no objects with $a<2$ AU and $q<1$ AU, though the $44 \mathrm{P}$ object in $n 1$ run spent $11.7 \mathrm{Myr}$ in such orbits. For series $n 2$ with RMVS3, we obtained one object with an initial orbit close to that of Comet 113P/Spitaler with relatively large values of probabilities of collisions with Earth and Venus. This object is responsible for $10 \%$ of the total collision probability with Earth for all $n 2$ objects, but most of the time spent by all these objects in orbits with $a<2$ AU and $q<1$ AU are due to this object. Though about a half of 30000 considered objects belong to series $n 2$, most of objects that spent a long time in Earth-crossing orbits with $Q<4.2 \mathrm{AU}$ belong to other series of runs.

After 40 Myr one considered object with an initial orbit close to that of Comet 88P/Howell (from $n 2$ RMVS3 runs) got $Q<3.5 \mathrm{AU}$, and it moved in orbits with $a=2.60-2.61 \mathrm{AU}, 1.7<q<2.2 \mathrm{AU}, 3.1<Q<3.5$ AU, eccentricity $e=0.2-0.3$, and inclination $i=5-10^{\circ}$ for $650 \mathrm{Myr}$. Another object (with an initial orbit close to that of Comet 94P/Russel 4) moved in orbits with $a=1.95-2.1 \mathrm{AU}, q>1.4 \mathrm{AU}, Q<2.6 \mathrm{AU}$, $e=0.2-0.3$, and $i=9-33^{\circ}$ for $8 \mathrm{Myr}$ (and it had $Q<3 \mathrm{AU}$ for $100 \mathrm{Myr}$ ). So JFCs can very rarely get typical asteroid orbits and move in them for Myrs. In our opinion, it can be possible that Comet 133P/Elst-Pizarro moving in a typical asteroidal orbit (Hsieh \& Jewitt 2006) was earlier a JFC and it circulated its orbit also due to non-gravitational forces. JFCs got typical asteroidal orbits less often than NEO orbits.

Levison et al. (2006) argued that our obtained orbits with $a \approx 1$ AU were due to the fact that collisions of objects with terrestrial planets were not taken into account in our runs and such orbits were caused by too close encounters of objects with planets which really result in collisions. Based on the orbital elements obtained in our runs, we can conclude that probabilities of collisions of migrating bodies with planets before bodies got orbits with $a<2$ AU were very small and the reason of the transformations of orbits was not caused by such close encounters of objects with the terrestrial planets that really resulted in collisions with the planets. Some real probabilities of collisions of bodies moving in orbits with $a<2 \mathrm{AU}$ with the terrestrial planets were only after bodies had already got such orbits and moved in them for tens or hundreds of Myr. Other 
scientists did not obtain the migration of JCOs into orbits with $a \approx 1$ AU because they considered other initial data. In series $n 2$ with 13500 objects, we also did not obtain orbits with $a \approx 1 \mathrm{AU}$ and obtained only two orbits with $a<2 \mathrm{AU}$ (the latter orbits were also obtained by Levison et al. 2006). For other series of runs, we paid particular attention to those initial data for which migrating objects could spend a long time inside Jupiter's orbit.

\section{Cometary objects in NEO orbits}

Based on the results of migration of JFCs with initial orbits close to the orbit of Comet P/1996 R2 Lagerkvist obtained by Ipatov \& Hahn (1999) (for these runs with about a hundred objects, there were no objects which spent a long time in Earth-crossing orbits), Ipatov $(1999,2001)$ found that $10-20 \%$ or more of all 1-km Earth-crossers could have come from the Edgeworth-Kuiper belt into Jupiter-crossing orbits. Using our results of the orbital evolution of 30000 JCOs and the results of migration of TNOs obtained by Duncan et al. (1995) and considering the total of $5 \times 10^{9} 1-\mathrm{km}$ TNOs with $30<a<50$ AU, Ipatov \& Mather (2003, 2004a,b) estimated the number of 1-km former TNOs in NEO orbits. The results of their runs testify in favor of at least one of these conclusions: (1) the portion of 1-km former TNOs among NEOs can exceed several tens of percents, (2) the number of TNOs migrating inside the solar system could be smaller by a factor of several than it was earlier considered, (3) most of 1-km former TNOs that had got NEO orbits disintegrated into mini-comets and dust during a smaller part of their dynamical lifetimes if these lifetimes are not small. All these three scenarios could take place. We consider that the role of disintegration may be more valuable and most of former comets that could move inside Jupiter's orbit for millions of years really were disintegrated. As the number of TNOs, their rate of migration inside the solar system, and lifetimes of former comets before their disruption are not well known, the estimates of the fraction of former TNOs among NEOs are very approximate.

Disintegrated comets could produce a lot of mini-comets and dust. Therefore there could be a lot of cometary dust among zodiacal particles, some of them were produced by high eccentricity comets (such as Comet $2 \mathrm{P} /$ Encke). The same conclusion about cometary dust was made by Ipatov et al. (2006a,b) based on analysis of spectra of the zodiacal light. Frank et al. $(1986 a, b)$ concluded that there is a large influx of small comets into the Earth's upper atmosphere.

It is known (Merline et al. 2002; Noll 2006; Pravec et al. 2006) that about $15 \%$ of NEOs and 2-3\% of main-belt asteroids are binaries. We can suppose that the fraction of NEO binaries is greater for those NEOs which are extinct comets than those for NEOs that came from the main asteroid belt. Comets more often split into smaller parts than asteroids, and probably there are former comets even among binary main-belt asteroids. Besides, if initial (before collisional destruction) small bodies were formed by compression of dust condensations, then the fraction of binary objects is greater for greater distances of the place of origin of bodies from the Sun (Ipatov 2004).

Comets are estimated to be active for $T_{a c t} \sim 10^{3}-10^{4} \mathrm{yr} . T_{a c t}$ is smaller for closer encounters with the Sun (Weissman et al. 2002), so for Comet 2P/Encke it is smaller than for other JFCs. If considered as material points, some former comets can move for tens or even hundreds of Myr in NEO orbits, so the number of extinct comets can exceed the number of active comets by several orders of magnitude. The mean time spent by Encke-type objects in Earth-crossing orbits was $\geqslant 0.4 \mathrm{Myr}$. This time corresponds to $\geqslant 40-400$ extinct comets of this type if we consider that Encke-type active comet is not an exceptional event in the history of the solar system. Note that the diameter of Comet 
$2 \mathrm{P} /$ Encke is about $5-10 \mathrm{~km}$, so the number of 1-km Earth-crossing extinct Encke-type comets can be greater by a factor of 25-100 than the above estimate for Encke-size comets and can exceed 1000 for such estimates. The rate of a cometary object decoupling from the Jupiter vicinity and transferring to a NEO-like orbit can be increased by a factor of several due to nongravitational effects (Harris \& Bailey 1998; Asher et al. 2001; Fernández $\&$ Gallardo 2002). The role of the Yarkovsky and YORP effects on dynamics of asteroids was summarized by Bottke et al. (2006).

Dynamical models of the NEO population considered by Bottke et al. (2002) allowed $6 \%$ of dead comets. From measured albedos, Fernández et al. (2001) concluded that the fraction of extinct comets among NEOs and unusual asteroids is significant (9\%). Rickman et al. (2001) and Jewitt \& Fernández (2001) considered that dark spectral classes that might include the ex-comets are severely under-represented and comets played an important and perhaps even dominant role among all km-size Earth impactors. Binzel \& Lupishko (2006) studied albedos and spectra of NEOs and concluded that $15 \pm 5 \%$ of the entire NEO population may be composed by extinct or dormant comets. Harris \& Bailey (1998) concluded that the number of cometary asteroids becomes comparable to the number of bodies injected from the main asteroid belt if one considers non-gravitational effects. Typical comets have larger rotation periods than typical NEOs (Binzel et al. 1992; Lupishko \& Lupishko 2001), but, while losing considerable portions of their masses, extinct comets can decrease these periods.

Our runs showed that if one observes former comets in NEO orbits, then most of them could have already moved in such orbits for millions (or at least hundreds of thousands) of years. Some former comets that have moved in typical NEO orbits for millions of years, and might have had multiple close encounters with the Sun, could have lost their mantles, which caused their low albedo, and so change their albedo (for most observed NEOs, the albedo is greater than that for comets (Fernández et al. 2001) and would look like typical asteroids.

\section{Probabilities of collisions of comets with planets}

The probability of a collision of one celestial body with a planet can be greater than the total probability for thousands of objects with almost the same initial orbit. All probabilities considered below were calculated for dynamical lifetimes of objects. A few JCOs (mentioned in Section 3) with the highest probabilities with planets were not included in the statistics presented below. For series $n 1$, the probability $P_{E}$ of a collision of an object with the Earth (during a dynamical lifetime of the object) was about $4.5 \times 10^{-6}$ and $4.8 \times 10^{-6}$ for BULSTO and RMVS3 runs, respectively (but for RMVS3 it is by an order of magnitude greater if we consider one more object with the highest probability). For series $n 2$, the mean value of $P_{E}$ was $\sim(10-15) \times 10^{-6}$ for BULSTO and RMVS3 runs.

Probabilities of collisions of JFCs with planets were different for different comets. The probability of a collision of Comet $10 \mathrm{P} /$ Tempel 2 with the Earth was $36 \times 10^{-6}$ and $22 \times 10^{-6}$ for BULSTO and RMVS3 runs, respectively $\left(P_{E}=140 \times 10^{-6}\right.$ if we include objects with high collision probabilities). For $2 \mathrm{P} /$ Encke runs, $P_{E}$ was relatively large: $\approx$ $(1-5) \times 10^{-4}$. For most other considered JFCs, $10^{-6} \leqslant P_{E} \leqslant 10^{-5}$. For Comets $22 \mathrm{P} /$ Kopff and $39 \mathrm{P} /$ Oterma, $P_{E} \approx(1-2) \times 10^{-6}$, and for Comets $9 \mathrm{P} /$ Tempel $1,28 \mathrm{P} /$ Neujmin 1 , and $44 \mathrm{P} /$ Reinmuth $2, P_{E} \approx(2-5) \times 10^{-6}$. The Bulirsh-Stoer method of integration and a symplectic method gave similar results. Values of $P_{E}$ were about $(0.5-2) \times 10^{-6}$ for $h t c$ runs, with greater values for smaller $q_{o}$. For $l p c$ runs, $P_{E}=0.6 \times 10^{-6}$ at $q_{o}=0.9 \mathrm{AU}$ and $P_{E}=0.25 \times 10^{-6}$ at $q_{o}=0.1$ AU. Dynamical lifetimes of some objects in htc and 
$l p c$ runs exceeded several Myr. Note that we considered collision probabilities for objects starting from different types of orbits, but a type of orbit (e.g., JFCs, HTCs, and LPCs) can change during the orbital evolution of objects.

The fraction of asteroids migrated from the 3:1 resonance with Jupiter that collided with the Earth was greater by a factor of several than that for the 5:2 resonance $\left(P_{E} \sim\right.$ $10^{-3}$ and $P_{E} \sim(1-3) \times 10^{-4}$, respectively). The probabilities of collisions with the Earth for resonant asteroids (per one object) were about two orders of magnitude greater than those for typical JFCs. The difference in $P_{E}$ for the asteroids and TNOs is greater than that for the asteroids and typical JFCs, as only about $1 / 3$ of TNOs that had leaved the trans-Neptunian belt reached Jupiter's orbit (Duncan et al. 1995). The present mass of the Edgeworth-Kuiper belt is considered to be about two orders of magnitude greater than that of the main asteroid belt. For dust particles started from comets and asteroids, $P_{E}$ was maximum for diameters $d \sim 100 \mu \mathrm{m}$ (Ipatov et al. 2004; Ipatov \& Mather 2006a,b). These maximum values of $P_{E}$ were usually (exclusive for $2 \mathrm{P} /$ Encke runs) greater at least by an order of magnitude than the values for parent comets.

The probabilities $P_{V}$ of collisions of JFCs and HTCs with Venus usually did not differ by more than a factor of 2 from those with Earth. For $2 \mathrm{P} /$ Encke runs, they were greater than those with Earth, but in most of other runs they were smaller. The probabilities $P_{M}$ of collisions of JFCs and HTCs with Mars usually were smaller by a factor of 3-6 (10 for the $2 \mathrm{P} /$ Encke runs) than those with Earth, i.e., Mars accreted more cometary bodies than Earth per unit of mass of the planet. For $l p c$ runs, the values of $P_{E}$ and $P_{V}$ can differ by a factor of 3 , and $P_{E} / P_{M} \sim 7-10$.

For most our runs, the probability $P_{J}$ of a collision of a JFC with Jupiter (during a dynamical lifetime of the comet) was $\sim 0.01$. Usually it was less than 0.03 , though it can be up to 0.06 in a single run. Due to resonances, the actual values of $P_{J}$ can be smaller than those in our runs. The mean time $T_{J}$ spent by objects in Jupiter-crossing orbits was $0.12 \mathrm{Myr}$ for $n 1$ runs. So the collision frequency of an object started from a JFC orbit and moving in a Jupiter-crossing orbit is about $10^{-7} \mathrm{yr}^{-1}$. Though $T_{J}$ can be a little greater for $2 \mathrm{P} /$ Encke runs than for $n 1$ and $n 2$ runs, and it can exceed 1 Myr for htc runs, $P_{J}$ was only about $5 \times 10^{-4}$ for some $2 \mathrm{P} /$ Encke and $h t c$ runs. In other $2 \mathrm{P} /$ Encke runs, $P_{J}$ can be greater or smaller by a factor of 20 than the above value. For $l p c$ runs, $P_{J}$ was smaller by an order of magnitude than that for $h t c$ runs though $T_{J}$ did not differ much.

Probabilities $P_{S}$ of collisions of objects from $n 1$ and $n 2$ runs with Saturn typically were smaller by an order of magnitude than those with Jupiter, and collision probabilities with Uranus and Neptune typically were smaller by three orders of magnitude than those with Jupiter. The ratio of probabilities of collisions of bodies with different giant planets, for a pair of planets can vary by more than an order of magnitude from run to run. As only a small fraction of comets collided with all planets during dynamical lifetimes of comets, the orbital evolution of comets for the considered model of material points was close to that for the model when comets collided with a planet are removed from integrations.

\section{Delivery of water and volatiles to planets}

Using $P_{E}=4 \times 10^{-6}$ (this value is smaller than the mean value of $P_{E}$ obtained in our runs for JFCs) and assuming that the total mass of planetesimals that ever crossed Jupiter's orbit is about $100 m_{\oplus}$ (Ipatov 1987, 1993), where $m_{\oplus}$ is the mass of the Earth, we obtain that the total mass of water delivered from the feeding zone of the giant planets to the Earth could be about the mass of water in Earth's oceans. We considered that the fraction $k_{w}$ of water in planetesimals equaled 0.5. For present comets $k_{w}<0.5$ (Jewitt 2004), but it is considered that $k_{w}$ could exceed 0.5 for planetesimals. The fraction of 
the mass of the planet delivered by JFCs and HTCs can be greater for Mars and Venus than that for the Earth. This larger mass fraction would result in relatively large ancient oceans on Mars and Venus. The conclusion that planetesimals from the zone of the giant planets could deliver all the water to the terrestrial oceans was also made by Ipatov (2001) and Marov \& Ipatov (2001) on the basis of runs by Ipatov \& Hahn (1999).

The above estimate of water delivery by cometary bodies to the Earth is greater than those by Morbidelli et al. (2000) and Levison et al. (2001), but is in accordance with the results by Chyba (1989) and Rickman et al. (2001). The larger value of $P_{E}$ we have calculated compared to those argued by Morbidelli et al. $(2000)\left(P_{E} \sim(1-3) \times 10^{-6}\right)$ and Levison et al. (2001) $\left(P_{E}=4 \times 10^{-7}\right)$ is caused by the fact that in our runs we considered other initial orbits and a larger number of JCOs. Levison et al. (2001) did not take into account the influence of the terrestrial planets, so probably that is why his values of $P_{E}$ are even smaller than those by Morbidelli et al. (2000). The latter authors used results of integrations of objects initially located beyond Jupiter's orbit. For 39P/Oterma runs $\left(a_{o}=7.25 \mathrm{AU}\right.$ and $\left.e_{o}=0.25\right)$, we obtained $P_{E}$ equal to $1.2 \times 10^{-6}$ and $2.5 \times 10^{-6}$ for BULSTO and RMVS3 runs, respectively. These values are in accordance with the values of $P_{E}$ obtained by Morbidelli et al. . Morbidelli et al. (2000) considered reasonable that about $50-100 m_{\oplus}$ of planetesimals primordially existed in the Jupiter-Saturn region and about $20-30 m_{\oplus}$ of planetesimals in the Uranus-Neptune region. We think that they considerably underestimated the mass of planetesimals in the Uranus-Neptune region.

Lunine $(2004,2006)$ concluded that possible sources of water for Earth are diverse, and include Mars-sized hydrated bodies in the asteroid belt, smaller "asteroidal" bodies, water adsorbed into dry silicate grains in the nebula, and comets. Lunine et al. (2003) considered most of the Earth's water as a product of collisions between the growing Earth and planet-sized "embryos" from the asteroid belt. Drake \& Campins (2006) noted that the key argument against an asteroidal source of Earth's water is that the O's isotopic composition of Earth's primitive upper mantle matches that of anhydrous ordinary chondrites, not hydrous carbonaceous chondrites. Kuchner et al. (2004) investigated the possibility that the Earth's ocean water originated as ice grains formed in a cold nebula, delivered to the Earth by drag forces from co-orbital nebular gas. Dust particles could also deliver water to the Earth from the feeding zone of the giant planets. Ipatov \& Mather (2006a,b) obtained that the probability of collisions of $10-100 \mu \mathrm{m}$ particles originated beyond Jupiter's orbit is about $(1-3) \times 10^{-4}$. Therefore the water in the terrestrial oceans $\left(2 \times 10^{-4} m_{\oplus}\right)$ can be delivered by particles (for the model without sublimation) which had contained $\sim m_{\oplus}$ of water when they had been located beyond Jupiter. So dust particles could also play some role in the delivery of water to the terrestrial planets during planet formation.

There is the deuterium/hydrogen paradox of Earth's oceans (the D/H ratio is different for oceans and comets), but Pavlov et al. (1999) suggested that solar wind-implanted hydrogen on interplanetary dust particles provided the necessary low-D/H component of Earth's water inventory, and Delsemme (1999) considered that most of the seawater was brought by the comets that originated in Jupiter's zone, where steam from the inner solar system condensed onto icy interstellar grains before they accreted into larger bodies. It is likely (Drake \& Campins 2006) that the $\mathrm{D} / \mathrm{H}$ and $\mathrm{Ar} / \mathrm{O}$ ratios measured in cometary comas and tails are not truly representative of cometary interiors.

Small bodies which collided with planets could deliver volatiles and organic/prebiotic compounds needed for life origin. Marov \& Ipatov (2005) concluded that dust particles could be most efficient in the delivery of organic or even biogenic matter to the Earth, because they experience substantially weaker heating when passing through the atmosphere (an excess heat is radiated effectively due to high total surface-to-mass ratio 
for dust particles). They assumed that life forms drastically different from the terrestrial analogs are unlikely to be found elsewhere in the solar system (if any), e.g., either extinct or extant life on Mars.

\section{Conclusions}

Some Jupiter-family comets can reach typical NEO orbits and remain there for millions of years. From the dynamical point of view (if comets didn't disintegrate) there could be (not 'must be') many (up to tens of percent) extinct comets among the NEOs, but, probably, many extinct comets disintegrated into mini-comets and dust during a smaller part of their dynamical lifetimes if these lifetimes were large. Disintegration of comets can provide a considerable fraction of cometary dust among the zodiacal dust particles. The probability of a collision of one object moving for a long time in Earth-crossing orbits, with the Earth could be greater than the sum of probabilities for thousands of other objects, even having similar initial orbits. Even without a contribution of such a few bodies, the probability of a collision of a former JFC (during its dynamical lifetime) with the Earth was greater than $4 \times 10^{-6}$. This probability is enough for delivery of all the water to Earth's oceans during formation of the giant planets. The ratios of probabilities of collisions of JFCs and HTCs with Venus and Mars to the mass of the planet usually were not smaller than that for Earth.

\section{References}

Asher, D.J., Bailey, M.E. \& Steel, D.I. 2001, in: M.Ya. Marov \& H. Rickman (eds.), Collisional Processes in the Solar System, ASSL 261, 121

Binzel, R.P., Xu, S., Bus, S.J. \& Bowell, E. 1992, Science 257, 779

Binzel, R.P., Lupishko, D. F., Di Martino, M., et al. 2002, in: W.F. Bottke Jr., A. Cellino, P. Paolicchi \& R. P. Binzel (eds.), Asteroids III (Tucson: Univ. of Arizona), p. 255

Binzel, R.P. \& Lupishko, D.F. 2006, in: D. Lazzaro, S. Ferraz-Mello \& J.A. Fernández (eds.), Asteroids, Comets, and Meteors, IAU Symposium 229 (Cambridge Univ. Press), p. 207

Bottke, W.F., Morbidelli, A., Jedicke, R., Petit, J.-M., Levison, H.F., Michel, P. \& Metcalfe, T.S. 2002, Icarus 156,399

Bottke, W.F., Vokrouhlický, D., Rubincam, D.P. \& Nesvorný, D. 2006, Annu. Rev. Earth Planet. Sci. 34, 157

Bulirsh, R. \& Stoer, J. 1966, Numer. Math. 8, 1

Chyba, C.F. 1989, Nature 343, 129

Delsemme, A.H. 1999, Planet. Space Sci. 47, 125

Drake, M. \& Campins, H. 2006, in: D. Lazzaro, S. Ferraz-Mello \& J.A. Fernández (eds.), Asteroids, Comets, and Meteors, IAU Symposium 229 (Cambridge Univ. Press), p. 381

Duncan, M.J., Levison, H.F. \& Budd, S.M. 1995, Astron. J. 110, 3073

Farinella, P., Gonczi, R., Froeschlé, Ch. \& Froeschlé, C. 1993, Icarus 101, 174

Fernández, J.A. \& Gallardo, T. 2002, Icarus 159, 358

Fernández, Y.R., Jewitt, D.C. \& Sheppard, S.S. 2001, ApJ 553, L197

Frank, L.A., Sigwarth, J.B. \& Graven, J.D. 1986a, Geophys. Res. Lett. 13, 303

Frank, L.A., Sigwarth, J.B. \& Graven, J.D. 1986b, Geophys. Res. Lett. 13, 307

Harris, N.W. \& Bailey, M.E. 1998, Mon. Not. R. Astron. Soc. 297, 1227

Hsieh, H.H. \& Jewitt, D. 2006, in: D. Lazzaro, S. Ferraz-Mello \& J.A. Fernández (eds.), Asteroids, Comets, and Meteors, IAU Symposium 229 (Cambridge Univ. Press), p. 425

Ipatov, S.I. 1987, Earth, Moon, \& Planets 39, 101

Ipatov, S.I. 1988, Soviet Astron. 32, 560

Ipatov, S.I. 1993, Solar Syst. Res. 27, 65

Ipatov, S.I. 1995, Solar Syst. Res. 29, 261

Ipatov, S.I. 1999, Celest. Mech. \& Dyn. Astr. 73, 107 
Ipatov, S.I. 2000, Migration of celestial bodies in the solar system, (Moscow: Editorial URSS Publishing Company) (in Russian)

Ipatov, S.I. 2001, Adv. Space Res. 28, 1107

Ipatov, S.I. 2002, in: B. Warmbein (ed.), Asteroids, comets, meteors, 2002, ESA SP-500, p. 371

Ipatov, S.I. 2004, in: S.S. Holt \& D. Deming (eds.), The Search for Other Worlds, AIP Conference Proceedings 713, p. 277

Ipatov, S.I. \& Hahn, G.J. 1999, Solar Syst. Res. 33, 487

Ipatov S.I. \& Mather J.C. 2003, Earth, Moon, 83 Planets 92, 89

Ipatov, S.I. \& Mather, J.C. 2004a, in: E. Belbruno, D. Folta \& P. Gurfil (eds.), Astrodynamics, Space Missions, and Chaos, Annals New York Acad. Sci. 1017, p. 46

Ipatov, S.I. \& Mather, J.C. 2004b, Adv. Space Res. 33, 1524

Ipatov, S.I. \& Mather, J.C. 2006a, Adv. Space Res. 37, 126

Ipatov, S.I. \& Mather, J.C. 2006b, in: H. Kruger \& A. Graps (eds.), Dust in Planetary Systems, in press, available from: http://arXiv.org/format/astro-ph/0606434

Ipatov, S.I., Mather, J.C. \& Taylor, P.A. 2004, in: E. Belbruno, D. Folta, \& P. Gurfil (eds.), Astrodynamics, Space Missions, and Chaos, Annals New York Acad. Sci. 1017, 66

Ipatov, S.I., Kutyrev, A.S., Madsen, G.J., Mather, J.C., Moseley, S.H. \& Reynolds, R.J. 2006a, 37th LPSC, \#1471

Ipatov, S.I., Kutyrev, A.S., Madsen, G.J., Mather, J.C., Moseley, S.H. \& Reynolds, R.J. 2006b, $A J$, submitted, available from: http://arXiv.org/format/astro-ph/0608141

Jewitt, D. 2004, in: M.C. Festou, H.U. Keller \& H.A. Weaver (eds.), Comets II (Tucson: Univ. Arizona Press), p. 659

Jewitt, D. \& Fernández, Y. 2001, in: M.Ya. Marov \& H. Rickman (eds.), Collisional Processes in the Solar System, ASSL 261, p. 143

Kuchner, M.J., Brown, M.E. \& Holman, M. 2002, Astron. J. 124, 1221

Kuchner, M.J., Youdin, A. \& Bate, M. 2004, Proc. of the Second TPF/Darwin Int. Conf., http://planetquest1.jpl.nasa.gov/TPFDarwinConf/confProceedings.cfm

Levison, H.F. \& Duncan, M.J. 1994, Icarus 108, 18

Levison, H.F. \& Duncan, M.J. 1997, Icarus 127, 13

Levison, H.F., Dones, L., Chapman, C.R., et al. 2001, Icarus 151, 286

Levison, H.F., Terrel, D., Wiegert, P.A., Dones, L. \& Duncan, M.J. 2006, Icarus 182, 161

Lunine, J.I. 2004, Proc. of the Second TPF/Darwin International Conference, http:// planetquest1.jpl.nasa.gov/TPFDarwinConf/confProceedings.cfm

Lunine, J.I., Chambers, J., Morbidelli, A. \& Leshin, L.A. 2003, Icarus 165, 1

Lunine, J.I. 2006, in: D.S. Lauretta \& H.Y. McSween Jr. (eds.), Meteorites and the Early Solar System II (Tucson: Univ. Arizona Press), p. 309

Lupishko, D.F. \& Lupishko, T.A. 2001, Solar Syst. Res. 35, 227

Marov, M.Ya. \& Ipatov, S.I. 2001, in: M.Ya. Marov \& H. Rickman (eds.), Collisional Processes in the Solar System, ASSL 261, p. 223

Marov, M.Ya. \& Ipatov, S.I. 2005, Solar Syst. Res. 39, 374

Merline, W.J., Weidenschilling, S.J., Durda, D.D., Margot, J.-L., Pravec, P. \& Storrs, A.D. 2002, in: W.F. Bottke Jr., A. Cellino, P. Paolicchi \& R.P. Binzel (eds.), Asteroids III (Tucson: Univ. of Arizona), p. 289

Morbidelli, A., Chambers, J., Lunine, J.I., Petit, J.-M., Robert, F., Valsecchi, G.B. \& Cyr, K.E. 2000, Meteorit. Planet. Sci. 35, 1309

Noll, K.S. 2006, in: D. Lazzaro, S. Ferraz-Mello \& J.A. Fernández (eds.), Asteroids, Comets, and Meteors, IAU Symposium 229 (Cambridge Univ. Press), p. 301.

Pavlov, A.A., Pavlov, A.K. \& Kasting, J.F. 1999, J. Geophys. Res. 104, 30725

Pravec, P., Scheirich, P., Kusnirak, P., et al. 2006, Icarus 181, 63

Rickman, H., Fernández, J.A., Tancredi, G. \& Licandro, J. 2001, in: M.Ya. Marov \& H. Rickman (eds.), Collisional Processes in the Solar System, ASSL 261, p. 131

Weissman, P.R., Bottke, W.F. Jr. \& Levison, H.F. 2002, in: W.F. Bottke Jr., A. Cellino, P. Paolicchi \& R.P. Binzel (eds.), Asteroids III (Tucson: Univ. of Arizona), p. 669

Wetherill, G.W. 1988, Icarus 76, 1 